\title{
Hematochezia before the First Feeding in a Newborn with Food Protein-Induced Enterocolitis Syndrome
}

\author{
Masanori Mizuno, Ph.D., M.D., ${ }^{1}$ Hiroshi Masaki, M.D., ${ }^{1}$ Ryoko Yoshinare, M.D., ${ }^{1}$ \\ Yujun Ito, M.D., ${ }^{1}$ Hideyuki Morita, M.D., ${ }^{2}$ and Hiroyuki Yoshio, Ph.D., M.D.
}

The prevalence and incidence of food protein-induced enterocolitis syndrome (FPIES) are clearly not known; its onset before first feeding at birth especially has been rarely reported. A female newborn was referred to our institution due to blood-stained diarrhea before her first feeding at birth. Examination of the stool with Wright-Giemsa staining on day 6 revealed numerous fecal eosinophils, including Charcot-Leyden crystals. Lymphocyte stimulation test (LST) against cow's milk protein also showed positive values on day 12. The hematochezia resolved immediately after starting intravenous nutrition. She was fed with breast milk and extensively hydrolyzed formula and discharged from hospital on day 49. FPIES was diagnosed based on these symptoms and data. Our case was thought to have acquired allergic enterocolitis after sensitization in her fetal period, which caused severe FPIES triggered by the first intake of cow's milk soon after birth. The patient with FPIES presents atypical clinical findings, which is likely to cause misdiagnosis and delay of appropriate treatment. Heightened awareness and increased attention may be necessary to diagnose FPIES, even soon after birth. Evaluating fecal eosinophils and LST, which may be difficult to perform in every clinical hospital, is thought to be useful for the detection of FPIES without oral food challenge.

KEYWORDS: Food protein-induced enterocolitis syndrome, hematochezia, fecal eosinophils, Charcot-Leyden crystals, Iymphocyte stimulation test

Food protein-induced enterocolitis syndrome (FPIES) is an uncommon condition and its pathophysiological features remain poorly understood. It is a non-immunoglobulin E ( $\mathrm{IgE}$ )-mediated disorder caused by gastrointestinal hypersensitivity. ${ }^{1,2}$ It is often difficult to diagnose FPIES rapidly and correctly because of its various atypical symptoms, resulting in unnecessary investigations and treatments. Delayed appropriate feeding and dietary advice often cause prolonged malabsorption. ${ }^{1,2}$ Most patients with FPIES show negative findings of skin prick tests, ${ }^{3,4}$ eosinocytes are often present in their mucous stools, ${ }^{5}$ and they react to the lymphocyte stimulation test (LST), ${ }^{6}$ which are helpful in diagnosing this disorder. Although hematochezia is ranked as one of the major FPIES primary symptoms, few neonatal cases presenting with it before

${ }^{1}$ Department of Pediatrics and Neonatology, St. Marianna University School of Medicine, Kawasaki; ${ }^{2}$ Department of Pediatrics, Gifu University Graduate School of Medicine, Gifu, Japan.

Address for correspondence and reprint requests: Masanori Mizuno, Ph.D., Department of Pediatrics, St. Marianna University School of Medicine, 2-16-1, Sugao, Miyamae-Ku, Kawasaki, Kanagawa, 216-8511 Japan (e-mail: m2.water-field@marianna-u.ac.jp).

Am J Perinatol Rep 2011;1:53-58. Copyright (C) 2011 by Thieme Medical Publishers, Inc., 333 Seventh Avenue, New York, NY 10001, USA. Tel: +1(212) 584-4662.

Received: December 2, 2011. Accepted after revision: March 29, 2011. Published online: June 9, 2011.

DOI: http://dx.doi.org/10.1055/s-0031-1280571.

ISSN 2157-6998. 

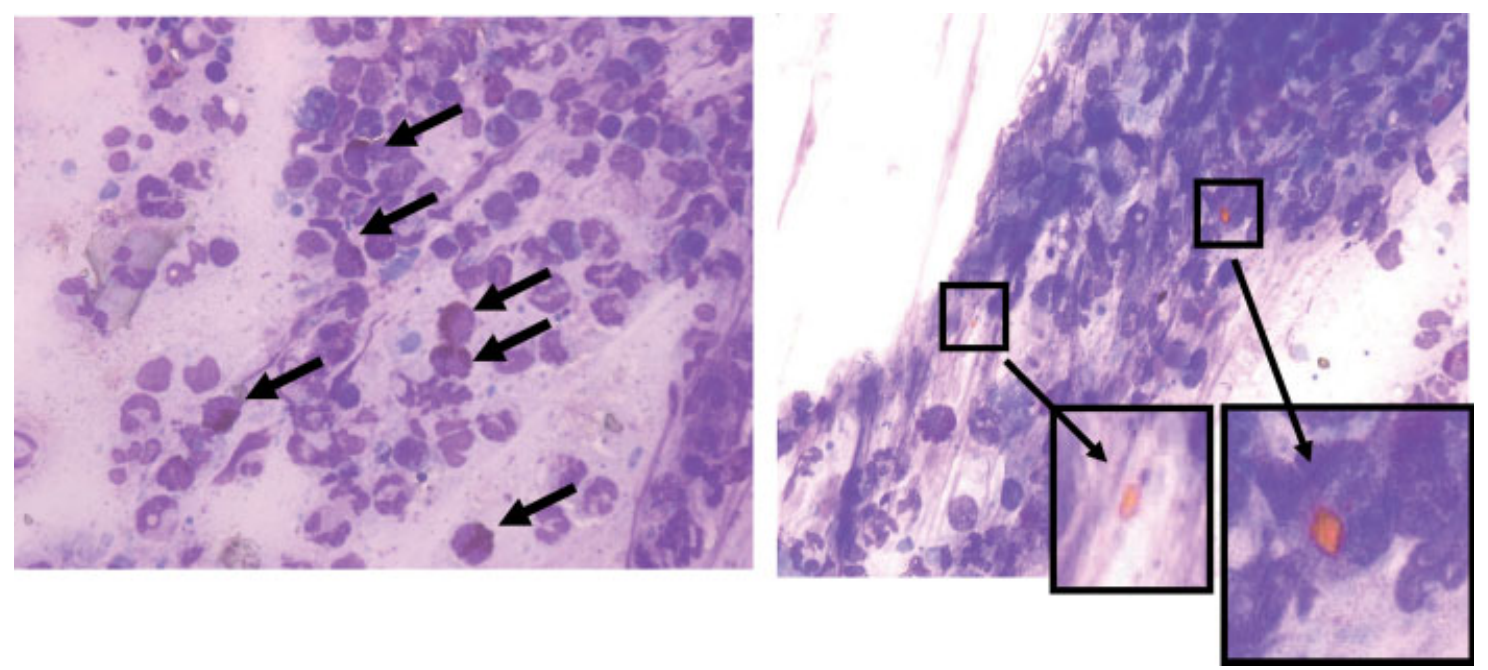

Figure 1 Wright-Giemsa staining of the mucous content of the stool showed numerous fecal eosinophils, including CharcotLeyden crystals that were aggregates of grains from collapsed eosinophils.

the first feeding have been reported, because other conditions in newborns more commonly cause hematochezia. ${ }^{7,8} \mathrm{We}$ report a rare case of a newborn with typical FPIES presenting with hematochezia before her first feeding, which was diagnosed based on the identification of fecal eosinocytes and a strong positive reaction to the LST.

\section{CASE REPORT}

The patient was a female neonate born to a 29-year-old Japanese primigravida, primipara woman. The vaginal delivery at 36 weeks of gestation was uneventful, and the infant weighed $2088 \mathrm{~g}$. No abnormalities during the gestational period were noted in either the patient or her mother. Apgar scores at 1 and 5 minute were both 9. She was referred to our institution on day 1 because bloodstained diarrhea occurred before her first feeding and continued thereafter. Despite bloody stool, she had been fed with formula, not with breast milk, several times until our hospital admission. Hematochezia continued until day 2 after birth, although she was administered 2 $\mathrm{mg}$ of vitamin $\mathrm{K}$ twice, immediately after birth and 14 hours later.

Physical examination on hospitalization revealed no abnormal signs, and initial blood laboratory data also showed normal results including coagulatory function tests. Laboratory examinations of blood, laryngeal, fecal, and cerebrospinal fluid cultures were all negative. $\mathrm{Ab}-$ dominal X-ray showed an air-rich stomach and gasless distal intestine. Empiric antibiotic (ampicillin and gentamicin) treatment for suspected infection was started due to a high C-reactive protein level $(15.6 \mathrm{mg} / \mathrm{dL})$ on day 2, although the white blood cell (WBC) count remained within the normal range. She was placed on intravenous nutrition alone, and the hematochezia resolved immediately.
Examination on day 6 showed a WBC of $30,000 / \mu \mathrm{L}$, with $8850 / \mu \mathrm{L}$ of eosinophils, and a diagnosis of allergy-induced enterocolitis was suspected. Wright-Giemsa staining of the mucous content of the stool was performed, and numerous fecal eosinophils were detected, including Charcot-Leyden crystals that were aggregates of grains from collapsed eosinophils (Fig. 1). When the LST was performed by using a blood sample collected on day 12 , the stimulation indices of casein and $\beta$-lactoglobulin were significantly elevated (7.16 and 7.12, respectively) and the patient's diagnosis was FPIES.

Antibiotics were discontinued on day7 because of poor response and clinical course. After starting intravenous nutrition without any enteral feeding from her admission, her clinical symptoms immediately began diminishing. Breast milk was given on day7 after her hematochezia had resolved. Although hematochezia did not recur, persistent diarrhea was encountered. A change to amino acid formula feeding on day 15 improved the diarrhea significantly, then her body weight began to increase. The change to extensively hydrolyzed formula feeding on day 28 did not cause diarrhea, and she gained weight well. Although anemia was detected with a hemoglobin level of $7.1 \mathrm{~g} / \mathrm{dL}$ and hematocrit of $21.5 \%$ on day 26, both increased after the administration of erythropoietin and ferrotherapy without transfusion. Because of diminishing hematochezia and diarrhea, she was finally discharged on a diet of extensively hydrolyzed formula with breast milk on day49 (Fig. 2).

\section{DISCUSSION}

Generally, FPIES is regarded as food protein stimulation of $\mathrm{T}$ cells in the gastrointestinal mucosa, ${ }^{1,2}$ although the mechanisms of occurrence have not been clarified. FPIES patients who present clinical symptoms at birth 


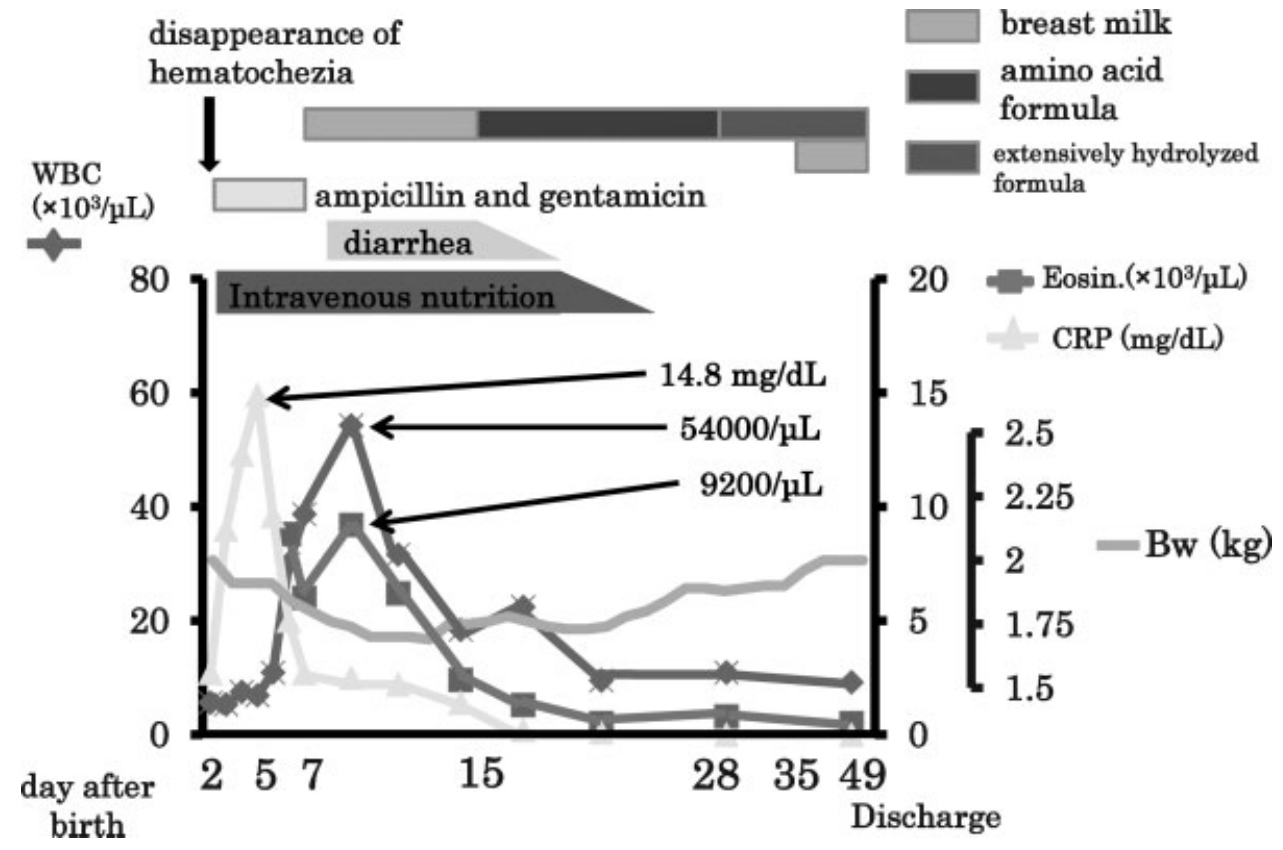

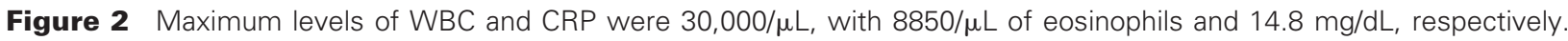
Amino acid formula and extensively hydrolyzed formula were started on the 15th and 28th neonatal day, respectively. The patient was discharged on a diet of extensively hydrolyzed formula with breast-feeding excluded on the 49th neonatal day. Bw, body weight; CRP, C-reactive protein; Eonin, eosinophils; WBC, white blood cell.

may acquire sensitization to milk protein in the fetal period as at least 10 days are required to form sensitization to food protein. ${ }^{9,10}$ Some FPIES patients present with intestinal inflammation referred to as "neonatal transient eosinophilic colitis" in the fetal period. ${ }^{11}$ Our case is thought to have acquired allergic enterocolitis after sensitization in the fetal period, which caused severe FPIES triggered by the first intake of cow's milk soon after birth.

Patients with FPIES show various atypical symptoms, and severe cases experience hypovolemic shock necessitating fluid resuscitation. Ikola and Jayasooriya et al reported cases mistakenly diagnosed as intussusception. ${ }^{7,8} \mathrm{We}$ did not administer a barium enema in our patient due to the fear of intestinal perforation. Thrombocytosis is often detected in FPIES, probably due to the effects of cytokines such as interleukin-6. ${ }^{1,12}$ Many reports on the relationship between cytokines and FPIES have been published. Douma et al reported that the stress-induced effects of epinephrine can shift platelets from the spleen into the bloodstream. ${ }^{13}$ Mori detected increased $\mathrm{CD}^{+}$cells in an infant with FPIES. $^{14} \mathrm{~A}$ recent review has reported increased tumor necrosis factor (TNF)- $\alpha$ in the stool of children with FPIES following a positive milk challenge. ${ }^{1,15}$ Heyman et al reported that TNF- $\alpha$ secreted by milk-specific $T$ lymphocytes increased intestinal permeability, leading to an influx of antigen into the submucosa and subsequent gastrointestinal inflammation. ${ }^{16}$ Chung et al found reduced transforming growth factor (TGF)- $\beta$ expression in the duodenum of FPIES patients, ${ }^{17}$ and Nowak-Wegrzyn and Muraro suggested that an imbalance in TGF- $\beta$ / TNF- $\alpha$ may play a role in the mechanism of FPIES development. ${ }^{1}$ Further studies are necessary to clarify the roles of these cytokine in FPIES.

Ingested food allergens induce initial gastrointestinal inflammation mediated by various cytokines such as TNF- $\alpha$ and interleukin-6. A delayed increase in the WBC, including high levels of eosinophils, is associated with the shift in platelets from the intestine to the circulation. ${ }^{13}$ These findings should prompt an examination of fecal eosinophils and performance of the LST in patients in whom FPIES is suspected.

A decrease in body temperature is often recognized as a presenting feature of FPIES. Mehr et al reported that $24 \%$ of FPIES patients had an initial presenting temperature of less than $36^{\circ} \mathrm{C}$ and that this finding was associated with significant circulatory volume loss. ${ }^{2}$ However, in our patient no significant decrease in body temperature was detected. Although we placed her in a closed incubator, no special measures to maintain body temperature were needed throughout the hospital admission period. Early intravenous hydration and the cessation of oral nutrition probably prevented fluid loss due to her diarrhea.

More than 90\% of FPIES patients have negative skin prick tests and undetectable food-specific $\mathrm{IgE}$ levels. ${ }^{3,4}$ An oral food challenge is necessary to diagnose FPIES, although most infants do not require the challenge test if they have a typical allergic reaction episode and their symptoms improve after elimination of the suspected food from their diet. As an oral food challenge 
is accompanied by the risk of anaphylactic shock, a safer, easier examination method is required. We emphasize that both the examination of fecal eosinophils and the LST results are useful in the diagnosis of FPIES. Endoscopic examination should not be performed routinely in suspected FPIES, because fecal eosinophil examination can be performed repeatedly, more easily, and more safely. Fecal eosinophils can be detected in normal neonatal stool in the first month, and the number of eosinophils should be evaluated thoroughly. CharcotLeyden crystals indicate the presence of numerous eosinophils and a diagnosis of FPIES.

LST results show high scores in FPIES, ${ }^{18}$ and thus the test may be more effective in detecting non$\mathrm{IgE}$-mediated allergic mechanisms, ${ }^{19}$ although few institutions can perform this test and about 1 week is needed before the results can be obtained. However, the LST should be performed when negative food-specific $\operatorname{IgE}$ is detected with peripheral or fecal eosinophilia.

Although an oral food challenge is necessary to diagnosis FPIES, it may cause severe allergic reactions of hypotension, hypothermia, and so on. ${ }^{2}$ The recommended age for performing an oral food challenge in infants suspected of having milk FPIES is more than 12 months, whereas the first soy challenge could be done between 6 and 8 months. ${ }^{20}$ Extensively hydrolyzed formula and amino acid formula should be used for alternative nutrition when a severe allergic reaction even to breast-feeding occurs. Sixty percent of FPEIS patients have both cow's milk and soy hypersensitivity, ${ }^{21}$ and rice is also a common cause of severe FPIES. ${ }^{22}$ Most FPIES patients lose hypersensitivity to milk and soy $\left(60 \%\right.$ and $25 \%$, respectively) by the age of 3 years. ${ }^{3,23}$ Hwang et al reported that milk and soy intolerance resolves at the age 20 and 14 months, respectively, in these patients. ${ }^{20}$

In conclusion, because FPIES patients present atypical clinical findings, heightened awareness and increased attention are important to diagnose FPIES, especially in neonates. Evaluating fecal eosinophils is simple, and although the LST may be difficult to perform, it allows the detection of non-IgE-mediated allergic reactions without oral food challenge.

\section{REFERENCES}

1. Nowak-Wegrzyn A, Muraro A. Food protein-induced enterocolitis syndrome. Curr Opin Allergy Clin Immunol 2009; 9:371-377

2. Mehr S, Kakakios A, Frith K, Kemp AS. Food proteininduced enterocolitis syndrome: 16-year experience. Pediatrics 2009;123:e459-e464

3. Sicherer SH, Eigenmann PA, Sampson HA. Clinical features of food protein-induced enterocolitis syndrome. J Pediatr 1998;133:214-219
4. Burks AW, Casteel HB, Fiedorek SC, Williams LW, Pumphrey CL. Prospective oral food challenge study of two soybean protein isolates in patients with possible milk or soy protein enterocolitis. Pediatr Allergy Immunol 1994;5: 40-45

5. Powell GK. Food protein-induced enterocolitis of infancy: differential diagnosis and management. Compr Ther 1986; 12:28-37

6. Motrich RD, Gottero C, Rezzonico C, Rezzonico C, Riera $\mathrm{CM}$, Rivero V. Cow's milk stimulated lymphocyte proliferation and TNFalpha secretion in hypersensitivity to cow's milk protein. Clin Immunol 2003;109:203-211

7. Ikola RA. Severe intestinal reaction following ingestion of rice. Am J Dis Child 1963;105:281-284

8. Jayasooriya S, Fox AT, Murch SH. Do not laparotomize food-protein-induced enterocolitis syndrome. Pediatr Emerg Care 2007;23:173-175

9. Ward CM, Geng L, Jyonouchi H. Fetal sensitization to cow's milk protein and wheat: cow's milk protein and wheatspecific TNF-alpha production by umbilical cord blood cells and subsequent decline of TNF-alpha production by peripheral blood mononuclear cells following dietary intervention. Pediatr Allergy Immunol 2007;18:276-280

10. Szépfalusi Z, Nentwich I, Gerstmayr M, et al. Prenatal allergen contact with milk proteins. Clin Exp Allergy 1997; 27:28-35

11. Ohtsuka Y, Shimizu T, Shoji H, et al. Neonatal transient eosinophilic colitis causes lower gastrointestinal bleeding in early infancy. J Pediatr Gastroenterol Nutr 2007;44: 501-505

12. Dame C, Sutor AH. Primary and secondary thrombocytosis in childhood. Br J Haematol 2005;129:165-177

13. Douma S, Zamboulis C, Karagiannis A, Sinakos Z. Effects of adrenaline infusion on serum thromboxane B2 and plasma $\beta$-thromboglobulin levels in hypertensive and normotensive subjects. Nouv Rev Fr Hematol 1992;34:73-78

14. Mori F, Barni S, Cianferoni A, Pucci N, de Martino M, Novembre E. Cytokine expression in CD3+ cells in an infant with food protein-induced enterocolitis syndrome (FPIES): case report. Clin Dev Immunol 2009;2009:679381; Epub

15. Benlounes N, Candalh C, Matarazzo P, Dupont C, Heyman M. The time-course of milk antigen-induced TNF-alpha secretion differs according to the clinical symptoms in children with cow's milk allergy. J Allergy Clin Immunol 1999; 104(4 Pt 1):863-869

16. Heyman M, Darmon N, Dupont C, et al. Mononuclear cells from infants allergic to cow's milk secrete tumor necrosis factor alpha, altering intestinal function. Gastroenterology 1994;106:1514-1523

17. Chung HL, Hwang JB, Kwon YD, Park MH, Shin WJ, Park JB. Deposition of eosinophil-granule major basic protein and expression of intercellular adhesion molecule-1 and vascular cell adhesion molecule- 1 in the mucosa of the small intestine in infants with cow's milk-sensitive enteropathy. J Allergy Clin Immunol 1999;103:1195-1201

18. Miyazawa T, Itahashi K, Imai T. Management of neonatal cow's milk allergy in high-risk neonates. Pediatr Int 2009;51: 544-547

19. Kondo N, Agata H, Fukutomi O, Motoyoshi F, Orii T. Lymphocyte responses to food antigens in patients with atopic dermatitis who are sensitive to foods. J Allergy Clin Immunol 1990;86:253-260 
20. Hwang JB, Sohn SM, Kim AS. Prospective follow-up oral food challenge in food protein-induced enterocolitis syndrome. Arch Dis Child 2009;94:425-428

21. Sicherer SH. Food protein-induced enterocolitis syndrome: case presentations and management lessons. J Allergy Clin Immunol 2005;115:149-156
22. Mehr SS, Kakakios AM, Kemp AS. Rice: a common and severe cause of food protein-induced enterocolitis syndrome. Arch Dis Child 2009;94:220-223

23. Nowak-Wegrzyn A, Sampson HA, Wood RA, Sicherer SH. Food protein-induced enterocolitis syndrome caused by solid food proteins. Pediatrics 2003;111(4 Pt 1):829-835 